TRANSACTIONS OF THE

AMERICAN MATHEMATICAL SOCIETY

Volume 176, February 1973

\title{
CODOMINANT DIMENSION OF RINGS AND MODULES
}

\author{
BY
}

\author{
GARY L. EERKES( $\left.{ }^{1}\right)$
}

\begin{abstract}
Expanding Nakayama's original concept of dominant dimension, Tachikawa, Müller and Kato have obtained a number of results pertaining to finite dimensional algebras and more generally, rings and their modules. The purpose of this paper is to introduce and examine a categorically dual notion, namely, codominant dimension. Special attention is given to the question of the relation between the codominant and dominant dimensions of a ring. In particular, we show that the two dimensions are equivalent for artinian rings. This follows from our main result that for a left perfect ring $R$ the dominant dimension of each projective left $R$-module is greater than or equal to $n$ if and only if the codominant dimension of each injective left $R$-module is greater than or equal to $n$. Finally, for computations, we consider generalized uniserial rings and show that the codominant dimension, or equivalently, dominant dimension, is a strict function of the ring's Kupisch sequence.
\end{abstract}

1. Preliminaries. Throughout this paper we shall assume that all rings are associative and have an identity and that all modules are unital. The category of all unital left $R$-modules of a ring $R$ will be denoted by ${ }_{R}$ M while $J$ will be used to indicate the Jacobson radical of $R$. Further, if $M$ is a module over $R$, $E_{R}(M)$ will denote the injective hull of $M$ and Soc $(M)$ the socle of $M$.

Let $M$ be an $R$-module with minimal projective resolution

$$
\text { .. } \longrightarrow P_{n} \stackrel{\phi_{n}}{\longrightarrow} \cdot \cdots P_{1} \stackrel{\phi_{1}}{\longrightarrow} M \longrightarrow 0 \text {, }
$$

i.e., $\phi_{i}: P_{i} \rightarrow \operatorname{Ker} \phi_{i-1}$ is a projective cover of $\operatorname{Ker} \phi_{i-1}$ for $i=1,2, \ldots$. The codominant dimension of $M$, denoted codom $\operatorname{dim} M$, is defined to be $\min \left\{i: P_{i+1}\right.$ is not injective $\}$ where we follow the convention of setting $\min \varnothing=\infty$.

So as to be assured of the existence of projective covers and therefore codominant dimension we will usually restrict our attention to perfect rings. In any case, whenever defined the codominant dimension of a module is well defined, as projective covers are unique to within an isomorphism.

Presented to the Society, January 17, 1972; received by the editors December 22, 1971. AMS (MOS) subject classifications (1970). Primary 16A60; Secondary 16A50, 16 A52.

Key words and phrases. Perfect rings, minimal injective cogenerator, minimal projective resolutions, injective projective modules, codominant and dominant dimensions, generalized uniserial rings, Kupisch sequences.

(1) This paper is taken from the author's doctoral dissertation written under the direction of Professor F. W. Anderson at the University of Oregon while the author was a National Science Foundation Graduate Fellow. The author wishes to express his appreciation to Professor Anderson for his guidance and encouragement.

Copyright $\odot$ 1973, American Mathematical Society 
Example 1.1. A ring with modules of arbitrary codominant dimension. Let $R$ be an algebra over a field $F$ with basis $\left\{e_{1}, e_{2}, \ldots\right\} \cup\left\{x_{1}, x_{2}, \ldots\right\} \cup\{1\}$ where multiplication is defined by $e_{i}^{2}=e_{i}, e_{i} x_{i} e_{i+1}=x_{i}, 1$ is a two-sided identity, all other products zero. Then the $R$-module $R e_{i} / J e_{i}$ has codominant dimension $i-1$. (This ring is given in [19, p. 379] as an example of a ring for which $\mathbb{M}_{R}$ possesses a noninjective cogenerator with no chain conditions on direct summands.)

We will need the following observation concerning codominant dimension's behavior over direct sums.

Proposition 1.2. Let $R$ be a left artinian ring. If ${ }_{R} M \cong \bigoplus \Sigma_{a \in \mathbf{\Omega}^{M}}$, then codom $\operatorname{dim} M=\min \left\{\right.$ codom $\left.\operatorname{dim} M_{a}: \alpha \in \Omega\right\}$. Further, for finite $\Omega$ we can relax artinian to perfect.

Proof. This is immediate, for if we take a direct sum of the minimal projective resolutions of the $M_{a}$ 's we obtain a minimal projective resolution of $M$.

Although we make no use of it, we also note a dual to a result of Tachikawa [21].

Proposition 1.3. If proj $\operatorname{dim} M<\operatorname{codom} \operatorname{dim} M$, then $M$ is injective and projective.

Proof. In such a case any minimal projective resolution of $M$ would necessarily be split exact.

Next we recall the definition of dominant dimension and note some results we need later.

Let $M$ be an $R$-module with minimal injective resolution

$$
0 \longrightarrow M \stackrel{\psi_{1}}{\longrightarrow} E_{1} \longrightarrow \cdot \cdots \longrightarrow E_{n} \stackrel{\psi_{n+1}}{\longrightarrow} \cdot \cdots,
$$

i.e., $E_{i} \cong E_{R}\left(E_{i-1} / \operatorname{Im} \psi_{i-1}\right)$. The dominant dimension of $M$, denoted $\operatorname{dom} \operatorname{dim} M$, is defined to be $\min \left\{i: E_{i+1}\right.$ is not projective $\}$. We define the left dominant dimension of $R$, denoted $l$-dom $\operatorname{dim} R$, to be $\operatorname{dom} \operatorname{dim}{ }_{R} R$.

As (1.2)'s dual, we have:

Proposition 1.4. Let $R$ be left noetberian and ${ }_{R} M \cong \bigoplus \Sigma_{a \in \Omega^{M}} a^{*}$. Then, $\operatorname{dom} \operatorname{dim} M=\min \left\{\operatorname{dom} \operatorname{dim} M_{a}: \alpha \in \Omega\right\}$. Also, $R$ can be arbitrary if $\Omega$ is finite.

Proof. By a result of Matlis [11, p. 514],

$$
E\left(\bigoplus \sum_{a \in \mathbf{\Omega}} M_{\alpha}\right) \cong \bigoplus \sum_{a \in \Omega} E\left(M_{\alpha}\right)
$$

Thus, by taking a direct sum of the minimal injective resolutions of the $M_{\alpha}$ 's we obtain a minimal injective resolution of $M$. 
This means, in the case of a left perfect ring, that $l$-dom $\operatorname{dim} R \geq n$ if and only if $\operatorname{dom} \operatorname{dim}{ }_{R} P \geq n$ for each indecomposable projective ${ }_{R} P$. For $R$ left artinian, we can drop indecomposable.

Proposition 1.5. Let $R$ be left artinian. Then the left dominant dimension of $R$ is greater than or equal to $n$ if and only if the dominant dimension of each projective left $R$-module is greater than or equal to $n$.

Proof. This follows from (1.4) and the characterization of projective left $R$-modules over left perfect rings as direct sums of indecomposable projectives, i.e., of $R e$ 's, $e$ a primitive idempotent.

To this point we have not defined the codominant dimension of the ring itself. Further, we observe that imitating the procedure used for dominant dimension, using the dimension of the left regular module ${ }_{R} R$, would be absurd. To motivate this promised but still unstated definition we make a few additional observations about its dual.

A projective generator ${ }_{R} P$ is a minimal projective generator for $R_{R}^{\text {Mn }}$ provided that for every other projective generator ${ }_{R} P^{\prime}$, there exists an epimorphism

$$
P^{\prime} \longrightarrow P \longrightarrow 0
$$

i.e., $P$ is isomorphic to a direct summand of every other projective generator.

Since ${ }_{R} R$ is always a projective generator for ${ }_{R}$ Ml it is immediate that if a minimal projective generator exists it must be isomorphic to $R e$ for some idempotent $e \in R$.

Example 1.6. A ring without minimal projective generators. Let $R=$ $\Pi_{i=1}^{\infty} M_{n}(D), D$ a division ring, and $e_{i j}^{n} \in M_{n}(D)$ with all entries zero excepting the $i, j$ th which is $1 \in D$. Then $R \cdot\left(e_{11}^{1}, \ldots, e_{11}^{n}, 1, \ldots, 1, \ldots\right)=P_{n}$ is a projective generator for $n=1,2, \cdots$. Suppose that ${ }_{R} P$ is a minimal projective generator. In this case, for each $n$ there exists an epimorphism $\phi_{n}: P_{n} \rightarrow P$. But ${ }_{R} P$ is a generator and ${ }_{R} R$ is cyclic, so there exists an epimorphism

$$
\text { S: } P^{m} \longrightarrow R \longrightarrow n
$$

for some $m$, where $P^{m}=\bigoplus \Sigma_{i=1}^{m} P$. Hence, for each $n$ there exists an epimorphism

$$
P_{n}^{m} \longrightarrow R \longrightarrow n
$$

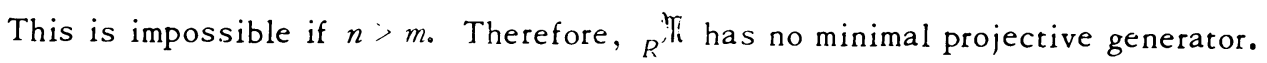

In the case of semiperfect rings, however, minimal projective generators do exist. In particular, if $e_{1}, \ldots, e_{n}$ is a basic set of idempotents for such a ring, then $R e_{1} \oplus \ldots \oplus R e_{n}$ is precisely such a module. Moreover, as we now show, minimal projective generators are unique to within an isomorphism for such rings. That is, if ${ }_{R} P$ and ${ }_{R} P^{\prime}$ are minimal projective generators for ${ }_{R} \mathbb{M}$, then 
$P \cong P^{\prime} \oplus P_{1}, P^{\prime} \cong P \oplus P_{2}$ and $P \cong P \oplus P_{1} \oplus P_{2}$. But then

$$
P / J P \cong\left(P \oplus P_{1} \oplus P_{2}\right) /\left(J P \oplus J P_{1} \oplus J P_{2}\right) \cong P / J P \oplus P_{1} / J P_{1} \oplus P_{2} / J P_{2}
$$

where $P / J P$ is artinian. Thus, $P_{1} / J P_{1} \oplus P_{2} / J P_{2}=0$ from which we have $P_{1}=$ $P_{2}=0$ and $P \cong P^{\prime}$.

We now note a categorical characterization of the left dominant dimension of $R$.

Proposition 1.7. If ${ }_{R} P$ is a minimal projective generator for ${ }_{R} \mathbb{M}$, then l-dom $\operatorname{dim} R=\operatorname{dom} \operatorname{dim}{ }_{R} P$.

Proof. This follows directly from (1.4).

Dualizing, we say that an injective cogenerator is a minimal injective cogenerator for ${ }_{R}$ 的 in case for every other injective cogenerator ${ }_{R} E$ there exists a monomorphism

$$
\cap \longrightarrow{ }_{R} U \longrightarrow{ }_{R} E
$$

i.e., $R_{R} U$ is isomorphic to a direct summand of every other injective cogenerator. We define the left codominant dimension of $R$, denoted $l$-codom $\operatorname{dim} R$, to be codom $\operatorname{dim}{ }_{R} U$ where ${ }_{R} U$ is a minimal injective cogenerator for $R_{R}^{M}$.

So as to settle questions concerning whether this definition is well defined, we give the following characterization of a minimal injective cogenerator.

Proposition 1.8. Let ${ }_{R} S=\bigoplus \Sigma_{a \in \mathbf{\Omega}} S_{a}$ where $\left\{S_{\alpha}: \alpha \in \Omega\right\}$ is a complete set of pairwise nonisomorphic representatives of the simple modules in $R$. Then, ${ }_{R} U \cong E_{R}(S)$ if and only if ${ }_{R} U$ is a minimal injective cogenerator for $R^{M}$.

Proof. It is well known (e.g., see Osofsky [19, p. 374]) that a module $R_{R}^{M}$ is a cogenerator if and only if ${ }_{R} M$ contains an injective hull of every simple module in $R^{\Re}$. Consequently, $E_{R}(S)$ is necessarily a minimal injective cogenerator.

Conversely, if ${ }_{R} U$ is a minimal injective cogenerator, then $E_{R}(S) \cong{ }_{R} U$ $\oplus N$ for some ${ }_{R} N$. Let this isomorphism be given by $\phi$. If $N \neq 0$, then Soc $(N)$ $\neq 0$ since $\phi(S)$ is essential in $U \oplus N$. This is impossible as $S$ is isomorphic to a submodule of $U$ while $S \cong \operatorname{Soc}\left(E_{R}(S)\right)$ has simple homogeneous components.

Corollary 1.9. Let $R$ be left perfect with basic set of idempotents $e_{1}, \cdots, e_{n}$. Then the left codominant dimension of $R$ is equal to the minimum of codom $\operatorname{dim} E\left(R e_{i} / J e_{i}\right)$ for $i=1, \cdots, n$.

Next, we note the existence of rings of arbitrary codominant dimension. 
Example 1.10. Let $R$ be the ring of upper triangular $n \times n$ matrices over a division ring. The Jacobson radical $J$ of $R$ is the ideal of all strictly upper triangular matrices in $R$. Further, if $e_{i j}$ denotes the matrix in $R$ all of whose entries are zero excepting for the $i, j$ th which is 1 , then

$$
R e_{22} \oplus \cdots \oplus R e_{n-1, n-1} \oplus R e_{n n} \oplus R e_{n n} / J e_{n n}
$$

is seen to be a minimal injective cogenerator for the ring $R / J^{2}$. It follows that $l$-codom $\operatorname{dim} R / J^{2}=$ codom $\operatorname{dim} R e_{n n} / J e_{n n}=n-1$.

Over a right perfect ring $R$ every left module hás a nonzero socle (see Bass [1, Theorem P]). It follows that every indecomposable injective left $R$-module is of the form $E_{R}(S)$ for ${ }_{R} S$ is a simple $R$-module. Hence, the left codominant dimension of a right perfect ring is greater than or equal to $n$ if and only if codom $\operatorname{dim}{ }_{R} E \geq n$ for each indecomposable injective left $R$-module ${ }_{R} E$. For a left artinian ring one can drop the indecomposable restriction in this equivalence.

Proposition 1.11. Let $R$ be left artinian. Then the left codominant dimension of $R$ is greater than or equal to $m$ if and only if the codominant dimension of each injective left $R$-module is greater than or equal to $m$.

Proof. Since $R$ is left artinian, for an arbitrary injective $R$-module ${ }_{R} E$ we have ${ }_{R} E \cong \bigoplus \Sigma_{\alpha \in \Omega} E\left(S_{\alpha}\right)$ where each ${ }_{R} S_{a}$ is simple. By (1.2),

codorn $\operatorname{dim}{ }_{R} E=\min \left\{\right.$ codom $\left.\operatorname{dim} E_{R}\left(S_{\alpha}\right): \alpha \in \Omega\right\}$

$$
\geq \min \left\{\text { codom } \operatorname{dim} E\left(R e_{i} / J e_{i}\right): i=1, \cdots, n\right\}=l-\text { codom } \operatorname{dim} R
$$

where $e_{1}, \ldots, e_{n}$ is a basic set of idempotents for $R$.

2. Relating codominant dimension to dominant dimension. We now give our main result, which reflects the dual nature of codominant dimension to dominant dimension. It might be noted that this generalizes a theorem of Fuller [4] which states that a left artinian ring is QF-3 if and only if each of its injective left modules has an injective projective cover.

Theorem 2.1. Let $R$ be left perfect. Then, codom $\operatorname{dim}_{R} E \geq n$ for each injective ${ }_{R} E$ if and only if $\operatorname{dom} \operatorname{dim}{ }_{R} P \geq n$ for each projective ${ }_{R} P$.

Proof. $(\Longrightarrow)$. We shall induct on $n$. Assuming that codom $\operatorname{dim}_{R} E \geq 1$ for every injective ${ }_{R}^{E}$ we let ${ }_{R} P$ be projective and consider 


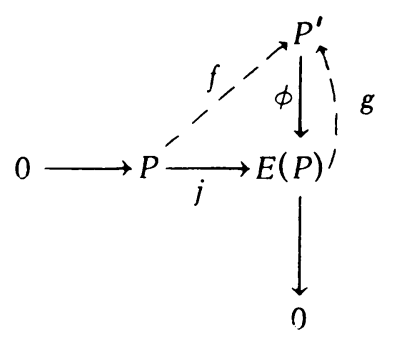

where $P^{\prime}$ is a projective cover of $E_{R}(P)$. Since $P$ is projective, the diagram can be completed with a homomorphism $f$ such that $\phi f=j$. But now, using the injectivity of $P^{\prime}$, there exists a homomorphism $g: E_{R}(P) \rightarrow P^{\prime}$ with $g j=f$. Furthermore, $g$ is monic since $f$ is and $\operatorname{Im} j$ is essential in $E_{R}(P)$. Hence, $E_{R}(P)$ is isomorphic to a direct summand of $P^{\prime}$ and is projective.

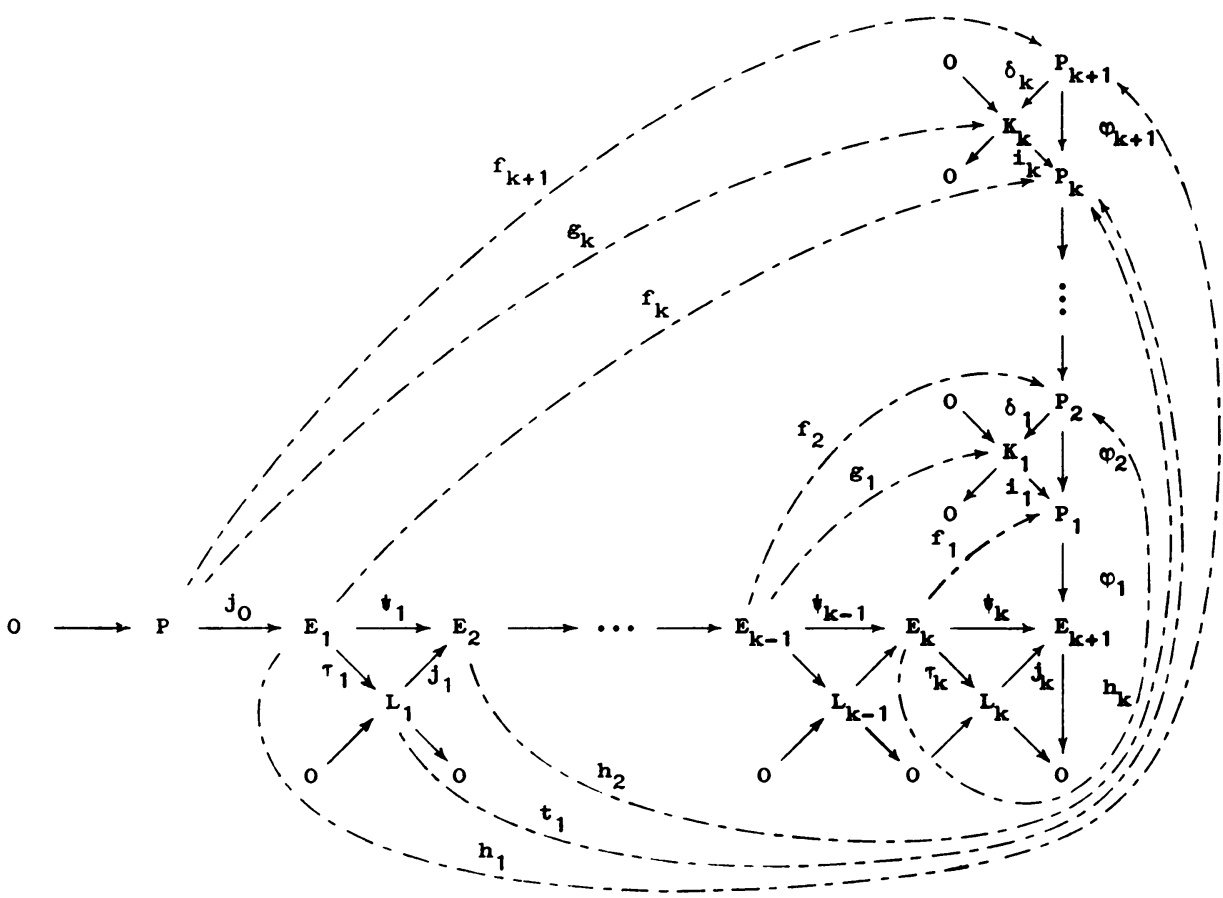

Figure 1

As the induction hypothesis we assume that codom $\operatorname{dim}{ }_{R} E \geq k+1$ for each injective ${ }_{R} E$ and that $\operatorname{dom} \operatorname{dim}{ }_{R} P^{\prime} \geq k$ for all projective ${ }_{R} P^{\prime}$. We then let ${ }_{R} P$ be projective and consider Figure 1 . In this diagram the row is a minimal injective resolution of ${ }_{R} P$ and the column is a minimal projective resolution of $E_{k+1}$, with the $L_{m}$ 's and $K_{m}$ 's being the naturally associated cokernels and 
kernels respectively. That is, $L_{m}=E_{m} / \operatorname{Im} j_{m-1}$ and $K_{m}=\operatorname{Ker} \phi_{m}$ while the maps $\tau_{m}, j_{m}, \delta_{m}$, and $i_{m}$ are the obvious ones.

To complete the proof we must show that the left $R$-module $E_{k+1}$ is projective. This will be done by reducing the situation to the setting where $n=1$. That is, we will establish the existence of a commutative diagram

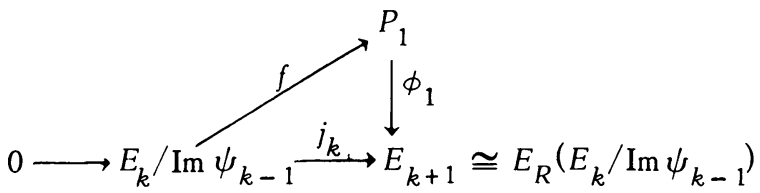

from which it will follow that $E_{k+1} \cong P_{1}$ is projective. In short, the remainder of the proof consists of constructing the map $f$ indicated above. This will be done in two steps.

Claim 1. There exist maps $f_{r}: E_{k+1-r} \rightarrow P_{r}, 1 \leq r \leq k+1, g_{r}: E_{k-r} \rightarrow K_{r}$, $1 \leq r \leq k$, such that $\delta_{r-1} f_{r}=g_{r-1}, i_{r} g_{r}=f_{r} \psi_{k-r}$ where we set $E_{0}=P, \psi_{0}=j_{0}$, $\delta_{0}=\phi_{1}$ and $g_{0}=\psi_{k}$.

Proof. Since $E_{k}$ is projective, we can complete

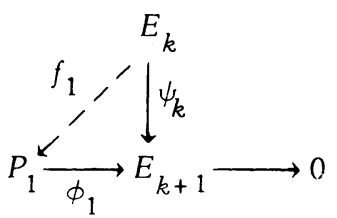

with a homomorphism $f_{1}$. Hitting the exact sequence

$$
0 \longrightarrow K_{1} \stackrel{i_{1}}{\longrightarrow} P_{1} \stackrel{\phi_{1}}{\longrightarrow} E_{k+1} \longrightarrow 0
$$

with the functor $\operatorname{Hom}_{R}\left(E_{k-1}\right.$, ), which is exact because by the induction hypothesis $E_{k-1}$ is projective, gives the exact sequence

$$
\begin{gathered}
0 \longrightarrow \operatorname{Hom}_{R}\left(E_{k-1}, K_{1}\right) \stackrel{\operatorname{Hom}\left(1, i_{1}\right)}{\longrightarrow} \operatorname{Hom}_{R}\left(E_{k-1}, P_{1}\right) \\
\stackrel{\operatorname{Hom}\left(1, \phi_{1}\right)}{\longrightarrow} \operatorname{Hon}_{R}\left(E_{k-1}, E_{k+1}\right) \longrightarrow 0 .
\end{gathered}
$$

Noting that $f_{1} \psi_{k-1} \in \operatorname{Hom}_{R}\left(E_{k-1}, P_{1}\right)$ while $\phi_{1} f_{1} \psi_{k-1}=\psi_{k} \psi_{k-1}=0$, we have that there exists $g_{1} \in \operatorname{Hom}_{R}\left(E_{k-1}, K_{1}\right)$ such that $i_{1} g_{1}=f_{1} \psi_{k-1}$. Now assume that $f_{r}, g_{r}$ have been defined for $1 \leq r \leq s \leq k$ such that $f_{r}: E_{k+1-r} \rightarrow P_{r}$, $g_{r}: E_{k-r} \rightarrow K_{r}$ and $\delta_{r-1} f_{r}=g_{r-1}, i_{r} g_{r}=f_{r} \psi_{k-r}$ where we set $E_{0}=P, \psi_{0}=j_{0}$, 
$\delta_{0}=\phi_{1}$ and $g_{0}=\psi_{k}$. Since $E_{k-s}$ is projective we can complete

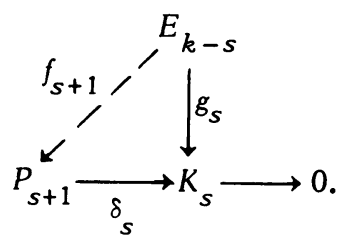

And for $s<k$, by applying $\operatorname{Hom}_{R}\left(E_{k-s-1}\right.$, ) to

we have the exact sequence

$$
0 \longrightarrow K_{s+1} \stackrel{i_{s+1}}{\longrightarrow} P_{s+1} \stackrel{\delta_{s}}{\longrightarrow} K_{s} \longrightarrow 0
$$

$$
\begin{aligned}
0 \longrightarrow \operatorname{Hom}_{R}\left(E_{k-s-1}, K_{s+1}\right) \stackrel{\operatorname{Hom}\left(1, i_{s+1}\right)}{\longrightarrow} \operatorname{Hom}_{R}\left(E_{k-s-1}, P_{s+1}\right) \\
\stackrel{\operatorname{Hom}\left(1, \delta_{s}\right)}{\longrightarrow} \operatorname{Hom}_{R}\left(E_{k-s-1}, K_{s}\right) \longrightarrow 0 .
\end{aligned}
$$

Since $f_{s+1} \psi_{k-s-1} \in \operatorname{Hom}_{R}\left(E_{k-s-1}, P_{s+1}\right)$ while

$$
i_{s} \delta_{s} f_{s+1} \psi_{k-s-1}=i_{s} g_{s} \psi_{k-s-1}=f_{s} \psi_{k-s} \psi_{k-s-1}=0 \text {, }
$$

where $i_{s}$ is monic, we have that $\delta_{s} f_{s+1} \psi_{k-s-1}=0$. But then there exists $g_{s+1}$ $\in \operatorname{Hom}_{R}\left(E_{k-s-1}, K_{s+1}\right)$ such that $i_{s+1} g_{s+1}=f_{s+1} \psi_{k-s-1}$. This completes the verification of our first claim. Observe that by combining the two relations involving the $f_{r}$ 's and $g_{r}$ 's we get $\phi_{r} f_{r}=f_{r-1} \psi_{k-r+1}$.

Claim 2. There exist maps $b_{r}: E_{r} \rightarrow P_{k+2-r^{\prime}} 1 \leq r \leq k$, and $t_{r}: L_{r} \rightarrow P_{k+1-r}$ $1 \leq r \leq k-1$, such that $b_{r} j_{r-1}=t_{r-1}, t_{r} \tau_{r}=f_{k+1-r}-\phi_{k+2-r} b_{r}$ where we set $t_{0}=f_{k+1}$.

Proof. Since $P_{k+1}$ is injective, we can complete

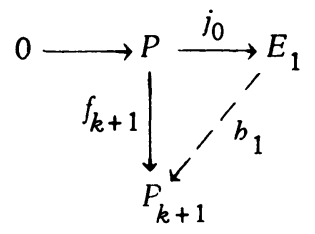

Also, since by hypothesis $P_{k}$ is injective, by applying $\operatorname{Hom}_{R}\left(, P_{k}\right)$ to

$$
0 \longrightarrow P \stackrel{j_{0}}{\longrightarrow} E_{1} \stackrel{\tau_{1}}{\longrightarrow} L_{1} \longrightarrow 0,
$$

we get the exact sequence

$0 \longrightarrow \operatorname{Hom}_{R}\left(L_{1}, P_{k}\right) \stackrel{\operatorname{Hom}\left(\tau_{1}, 1\right)}{\longrightarrow} \operatorname{Hom}_{R}\left(E_{1}, P_{k}\right) \stackrel{\operatorname{Hom}\left(j_{0}, 1\right)}{\longrightarrow} \operatorname{Hom}_{R}\left(P, P_{k}\right) \longrightarrow 0$. 
Further, by observing that $f_{k}-\phi_{k+1} b_{1} \in \operatorname{Hom}_{R}\left(E_{1}, P_{k}\right)$ and that

$$
\left(f_{k}-\phi_{k+1} b_{1}\right) j_{0}=f_{k} j_{0}-\psi_{k+1} f_{k+1}=f_{k} j_{0}-f_{k} j_{0}=0
$$

we have a homomorphism $t_{1} \in \operatorname{Hom}_{R}\left(L_{1}, P_{k}\right)$ such that $t_{1} \tau_{1}=f_{k}-\phi_{k+1} b_{1}$. Now by assuming that $b_{r}, t_{r}$ have been defined for $1 \leq r \leq s \leq k-1$ such that $b_{r}$ : $E_{r} \rightarrow P_{k+2-r^{\prime}} t_{r}: L_{r} \rightarrow P_{k+1-r^{\prime}} b_{r} j_{r-1}=t_{r-1}$, and $t_{r r} \tau_{r}=f_{k+1-r}-\phi_{k+2-r} b_{r}$ (where we set $t_{0}=f_{k+1}$ ), we define $b_{s+1}$ by completing

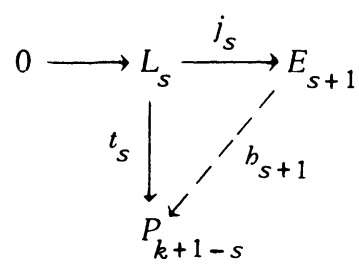

On the other hand, if $s \leq k-2$ we define $t_{s+1}$ by first hitting

$$
0 \longrightarrow L_{s} \stackrel{j_{s}}{\longrightarrow} E_{s+1} \stackrel{\tau_{s+1}}{\longrightarrow} L_{s+1} \longrightarrow 0
$$

with the exact functor $\operatorname{Hom}_{R}\left(, P_{k-s}\right)$, to get

$$
\begin{aligned}
0 \longrightarrow & \operatorname{Hom}\left(L_{s+1}, P_{k-s}\right) \stackrel{\operatorname{iom}\left(\tau_{s+1}, 1\right)}{\longrightarrow} \operatorname{Hoin}\left(E_{s+1}, P_{k-s}\right) \\
& \stackrel{\operatorname{Hom}\left(j_{s}, 1\right)}{\longrightarrow} \operatorname{Hom}\left(L_{s}, P_{k-s}\right) \longrightarrow 0
\end{aligned}
$$

and then observing that $f_{k-s}-\phi_{k+1-s} b_{s+1} \in \operatorname{Hom}_{R}\left(E_{s+1}, P_{k-s}\right)$ and that

$$
\begin{aligned}
& \left(f_{k-s}-\phi_{k+1-s} b_{s+1}\right) j_{s}{ }^{\top} s=f_{k-s} \psi_{s}-\phi_{k+1-s} t^{\tau}{ }^{\tau} s \\
& \quad=f_{k-s} \psi_{s}-\phi_{k+1-s}\left(f_{k+1-s}-\phi_{k+2-s} b_{s}\right)=f_{k-s} \psi_{s}-\phi_{k+1-s} f_{k+1-s} \\
& \quad=f_{k-s} \psi_{s}-f_{k-s} \psi_{s}=0
\end{aligned}
$$

where $\tau_{s}$ is epic. Thus, $\left(f_{k-s}-\phi_{k+1-s} b_{s+1}\right) j_{s}=0$ and so there exists $t_{s+1} \epsilon$ Hom $_{R}\left(L_{s+1}, P_{k-s}\right)$ such that $t_{s+1} \tau_{s+1}=f_{k-s}-\phi_{k+1-s} b_{s+1}$. This completes the verification of our second claim.

We are now ready to construct the map $f$ indicated in the first part of the proof. We first consider $f_{1}-\phi_{2} b_{k}: E_{k} \rightarrow P_{1}$. We note that $\operatorname{Im} j_{k-1} \leq$ $\operatorname{Ker}\left(f_{1}-\phi_{2} b_{k}\right)$ since, as seen in the proof of the second claim, $\left(f_{1}-\phi_{2} b_{k}\right) j_{k-1}$ $=0$. On the other hand, if $\left(f_{1}-\phi_{2} b_{k}\right)(x)=0$ for some $x \in E_{k}$, then $\psi_{k}(x)=$ $\phi_{1} \phi_{2} b_{k}(x)=0$, i.e., $x \in \operatorname{Ker} \psi_{k}=\operatorname{Im} j_{k-1}$. The refore, $\operatorname{Ker}\left(f_{1}-\phi_{2} b_{2}\right)=\operatorname{Im} j_{k-1}$. But then $f_{1}-\phi_{2} h_{k}$ induces a map $E_{k} / \operatorname{Im} j_{k-1} \rightarrow P_{1}$. This is our map $f$. Now consider 


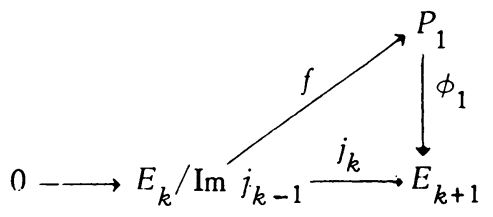

It is easy to check that this diagram is commutative. This is exactly the situation we had when $n=1$. It follows that $E_{k+1}$ is isomorphic to $P_{1}$ and so, is projective. This completes the proof of the sufficiency part of (2.1). Observe that since $E_{k+1}$ is projective the column in Figure 1 is trivial for all but $E_{k+1}$ and $P_{1}$.

$(\Longleftarrow)$. This half of the proof is the dual of the first.

Corollary 2.2. Let $R$ be a left artinian ring. Then, l-dom $\operatorname{dim} R \geq n$ if and only if $l$-codom $\operatorname{dim} R \geq n$.

Proof. This follows immediately from (1.5) and (1.11).

Corollary 2.3. If $R$ is artinian, then the left codominant dimension of $R$ is equal to the right codominant dimension of $R$.

Proof. Müller [14, Theorem 10] has proved that for $R$ perfect, $r$-dom $\operatorname{dim} R>0$ and $l$-dom $\operatorname{dim} R>0$ implies $l$-dom $\operatorname{dim} R=r-\operatorname{dom} \operatorname{dim} R$. On the other hand, Harada [5] has shown that if $R$ is artinian, then $l-\operatorname{dom} \operatorname{dim} R>0$ if and only if $r$-dom $\operatorname{dim} R>0$.

Theorem 2.4. Let $R$ be semiperfect and left noetberian. Then the following are equivalent:

(a) $l$-dom $\operatorname{dim} R \geq n$;

(b) $\operatorname{dom} \operatorname{dim}{ }_{R} P \geq n$ for each finitely generated projective ${ }_{R} P$;

(c) $\operatorname{dom} \operatorname{dim}_{R} P \geq n$ for each finitely generated indecomposable projective ${ }_{R}^{P}$;

(d) codom $\operatorname{dim}{ }_{R} Q \geq n$ for each finitely generated injective ${ }_{R} Q$ and each finitely generated projective ${ }_{R} P$ bas a minimum injective resolution whose first $n$ terms are finitely generated;

(e) codom $\operatorname{dim}{ }_{R} Q \geq n$ for each finitely generated indecomposable injective ${ }_{R} Q$ and each finitely generated indecomposable projective ${ }_{R} P$ bas a minimum injective resolution whose first $n$ terms are finitely generated.

Proof. By (1.2) and the characterization of finitely generated projective modules over semiperfect rings, we have that (a), (b) and (c) are equivalent. The equivalence of (d) and (e) is immediate from (1.4).

To show that (b) is sufficient for (d) it will suffice to indicate how the suf- 
ficiency proof of $(2.1)$ can be applied. Le ${ }_{R} Q$ be finitely generated injective with minimum projective resolution

$$
\text { •. } \longrightarrow P_{n} \longrightarrow \cdot \cdot \longrightarrow P_{1} \longrightarrow Q \longrightarrow 0 .
$$

The module $P_{1}$ is finitely generated since ${ }_{R} Q$ is. Further, the kernel of $P_{1} \rightarrow Q$ is finitely generated since $R$ is left noetherian. But then $P_{2}$ is finitely generated. By induction, $P_{1}, \cdots, P_{n}$ are all finitely generated. So, by hypothesis $\operatorname{dom} \operatorname{dim} P_{n} \geq n$ and it is straightforward to see that the same inductive proof as used in the sufficiency proof of (2.1) may be applied to obtain codom $\operatorname{dim}{ }_{R} Q \geq n$.

For ${ }_{R} P$ finitely generated projective with minimum injective resolution

$$
0 \longrightarrow P \longrightarrow E_{1} \longrightarrow \cdot \cdot \longrightarrow E_{n} \longrightarrow \cdot \cdot
$$

we note that (see Müller [14, Lemma 1]) $E_{1}$ is finitely generated by a result of Faith and Walker [2, Proposition 2.4] since it is projective. By induction we get that $E_{1}, \cdots, E_{n}$ are all finitely generated.

Finally, we assume (d) and let ${ }_{R} P$ be finitely generated with minimum injective resolution

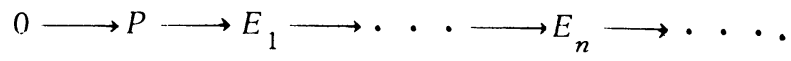

Since $E_{n}$ is finitely generated by hypothesis, codom $\operatorname{dim} E_{n} \geq \dot{n}$ and one shows inductively as in the necessity proof of (2.1) that $E_{n}$ is projective. Hence, (b) is equivalent to (d).

3. Codominant dimension of generalized uniserial rings. Recall that a generalized uniserial ring $R$ is an artinian ring for which each primitive one-sided ideal (i.e., each $R e$ and $e R$ where $e$ is a primitive idempotent) has a unique composition series. In the case where the ring is indecomposable, one can relate these indecomposable projectives in the following manner.

Theorem 3.1 (Kupisch [10]). If $R$ is an indecomposable generalized uni. serial ring, then any basic set of primitive idempotents $e_{1}, \cdots, e_{n}$ for $R$ can be indexed so that

(a) $R e_{i} / J e_{i} \cong J e_{i+1} / J^{2} e_{i+1}$, for $i=1, \cdots, n-1 ;$ and $R e_{n} / J e_{n} \cong J e_{1} / J^{2} e_{1}$ unless $\mathrm{Je} e_{1}=0$;

(b) the composition length of $R e_{i}=c\left(R e_{i}\right) \geq 2$, for $i=2, \cdots, n$;

(c) $c\left(R e_{i+1}\right) \leq c\left(R e_{i}\right)+1$ for $i=1, \cdots, n-1$;

(d) $c\left(R e_{1}\right) \leq c\left(R e_{n}\right)+1$.

In this setting, $R e_{1}, \cdots, R e_{n}$ is called a left Kupisch series for $R$ and $c_{1}=c\left(R e_{1}\right), \cdots, c_{n}=c\left(R e_{n}\right)$ is referred to as the corresponding admissible 
Kupisch sequence for $R$. Observe that in general $c_{1}, \cdots, c_{n}$ is unique to within an $n$-cycle. We say that $R e_{i}$ is a chain end provided $c\left(R e_{[i+1]}\right) \leq c\left(R e_{i}\right)$, where [i] denotes the least strictly positive remainder of $i$ modulo $n$. It can be shown that the chain ends are precisely the indecomposable injective projective $R$-modules (see Jans [6, p. 1107]).

In light of the work done by Kupisch, Murase, and Fuller with a generalized uniserial ring's Kupisch series, it is natural to attempt to compute the codominant dimension (or equivalently the dominant dimension) of such a ring from its Kupisch sequence. Indeed, Fuller showed how this sequence of integers characterizes the global dimension of the ring. In this section we prove a similar characterization for codominant dimension.

First we state a somewhat technical but useful result due to Kupisch [10] and Fuller [3].

Lemma 3.2. Let $R$ be an indecomposable generalized uniserial ring with Kupisch series $R e_{1}, \cdots, R e_{n}$ and $J^{k} e_{i} \neq 0$. Then,

(a) $J^{k} e_{i} \cong R e_{[i-k]} / J^{c i-k} \cdot e_{[i-k]}$;

(b) $J^{k} e_{i} / J^{k+1} e_{i} \cong R e_{[i-k]} / J e_{[i-k]}$;

(c) $E\left(R e_{i} / J e_{i}\right) \cong R e_{\left[i+d_{i}-1\right]} / J^{d_{i}} \cdot e_{\left[i+d_{i}-1\right]}$ where $d_{i}=c\left(e_{i} R\right)$.

Proposition 3.3. The codominant dimension of an indecomposable generalized uniserial ring is completely determined by the ring's Kupisch sequence.

Proof. Let $R e_{1}, \cdots, R e_{n}$ be a Kupisch series for $R$ and $c_{1}, \cdots, c_{n}$ its corresponding Kupisch sequence of integers. In light of (1.9) it will suffice to show that codom $\operatorname{dim} E\left(R e_{i} / J e_{i}\right)$ is completely determined by $c_{1}, \cdots, c_{n}$ for $i=1, \cdots, n$.

With this in mind, we fix $i$, set $\Omega_{i}=\inf \left\{j: R e_{k_{j}}\right.$ is not a chain end $\}$, and inductively define two sequences $\left\{s_{j}\right\},\left\{k_{j}\right\}$ by setting

$$
s_{0}=c\left(e_{i} R\right), \quad k_{0}=\left[i+s_{0}-1\right]
$$

and

$$
s_{j}=c_{k_{j-1}}-s_{j-1}, \quad k_{j}=\left[k_{j-1}-s_{j-1}\right]
$$

We shall show that codom $\operatorname{dim} E\left(R e_{i} / \mathrm{J} e_{i}\right)$ is equal to $\infty$ or $\Omega_{i}$ depending on whether or not $s_{j} \leq 0$ for some $j \leq \Omega_{i}$.

Fix $m<\Omega_{i}$. By (3.2)

$$
E_{R}\left(R e_{i} / J e_{i}\right) \cong R e_{k_{0}} / J^{s} 0 \cdot e_{k_{0}}
$$

and 


$$
J^{s} \cdot e_{k_{j}} \cong R e_{k_{j+1}} / J^{s+1} \cdot e_{k_{j+1}}
$$

provided $s_{j+1}>0$. Moreover, in this case $R e_{k_{j+1}}$ is a projective cover for $J^{s} j \cdot e_{k_{j}}$ Hence, assuming that $s_{j}>0$ for $j=1,2, \cdots, m$,
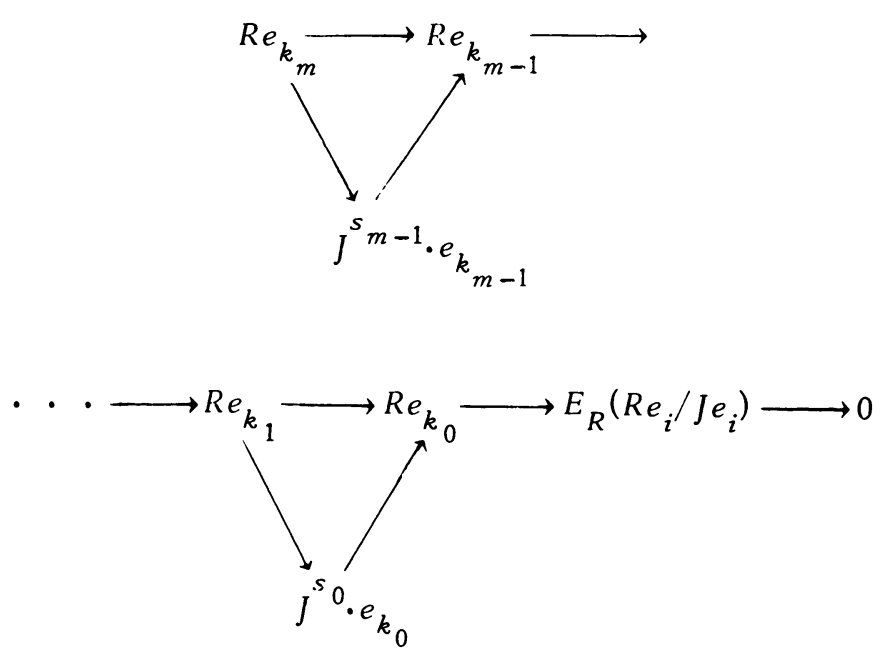

affords the first $m+1$ terms of a minimal projective resolution of $E_{R}\left(R e_{i} / J e_{i}\right)$. Since $R e_{k_{j}}$ is a chain end for $j=0,1, \cdots, m$ and so, injective, codom $\operatorname{dim} E_{R}\left(R e_{i} / J e_{i}\right) \geq m+1$. Consequently, codom $\operatorname{dim} E_{R}\left(R e_{i} / J e_{i}\right) \geq \Omega_{i}$.

Now if codom $\operatorname{dim} E_{R}\left(R e_{i} / \mathrm{J} e_{i}\right)>\Omega_{i}$ and $s_{j}>0$ for each $j \leq \Omega_{i}$, then by considering the above minimal projective resolution of $E_{R}\left(R e_{i} / \mathrm{J} e_{i}\right)$ we see that $R e_{k_{\Omega_{i}}}$ must be injective and hence, a chain end. This is impossible, however, so we conclude that codom $\operatorname{dim} E_{R}\left(R e_{i} / J e_{i}\right)=\Omega_{i}$.

Finally, if $s_{j} \leq 0$ for some $j \leq \Omega_{i}$, then it is immediate that codom $\operatorname{dim} E_{R}\left(R e_{i} / \mathrm{Je}{ }_{i}\right)=\infty$.

Lemma 3.4. Let $R_{1}, R_{2}$ be left perfect rings and $M_{1} \epsilon_{R_{1}} M, M_{2} \epsilon_{R_{2}}$ M. Then $M_{1}$ and $M_{2}$ are naturally $R_{1} \oplus R_{2}$-modules and

$$
\text { codom } \operatorname{dim} R_{1} \oplus R_{2} M_{1} \oplus M_{2}=\min _{i=1,2} \operatorname{codom~dim}{ }_{R_{i}}{ }^{M_{i}} \text {. }
$$

Proof. Let

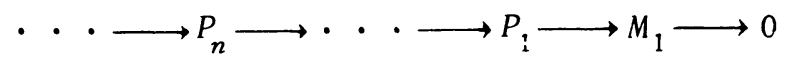

and

$$
\text { . . } \longrightarrow P_{n}^{\prime} \longrightarrow \cdot \cdot \longrightarrow P_{1}^{\prime} \longrightarrow M_{2} \longrightarrow 0
$$

be minimal projective resolutions of $R_{1} M_{1}$ and $R_{2} M_{2}$ respectively. Then, 
. . $\longrightarrow P_{n} \oplus P_{n}^{\prime} \longrightarrow \cdot \cdot \longrightarrow P_{1} \oplus P_{1}^{\prime} \longrightarrow M_{1} \oplus M_{2} \longrightarrow 0$

is a minimal projective resolution of $M_{1} \oplus M_{2} \epsilon_{R_{1} \oplus R_{2}}$ M, where each summand is now considered as an $R_{1} \oplus R_{2}$-module. Further, $P_{i} \oplus P_{i}^{\prime}$ is $R_{1} \oplus R_{2}$ injective if and only if $P_{i}$ is $R_{1}$ injective and $P_{i}^{\prime}$ is $R_{2}$ injective.

Proposition 3.5. Let $R_{1}$ and $R_{2}$ be left perfect rings. Then, l-codom $\operatorname{dim} R_{1} \oplus R_{2}=\min _{i=1,2} l$-codom $\operatorname{dim} R_{i}$.

Proof. Each simple left $R_{1} \oplus R_{2}$-module can be obtained from a simple $R_{i}$-module, say $R_{i} S$, for either $i=1$, or 2 , by considering $S$ as an $R_{1} \oplus R_{2}$-module. Also, $E_{R_{1} \oplus R_{2}}(S) \cong R_{1} \oplus R_{2}\left(E_{R_{i}}(S)\right)$ where we consider $E_{R_{i}}(S)$ as an $R_{1} \oplus R_{2}$-module. But then, by (3.4)

$$
\text { codom } \operatorname{dim} E_{R_{1} \oplus R_{2}}(S)=\text { codom } \operatorname{dim} E_{R_{i}}(S)
$$

The desired result now follows from (1.9).

Corollary 3.6. The codominant dimension of a generalized uniserial ring is completely determined by the admissible sequences of its indecomposable direct summands.

\section{REFERENCES}

1. H. Bass, Finitistic dimension and a homological generalization of semi-primary rings, Trans. Amer. Math. Soc. 95 (1960), 466-488. MR 28 \#1212.

2. C. Faith and E. A. Walker, Direct-sum representations of injective modules, J. Algebra 5 (1967), 203-221. MR 34 \#7575.

3. K. R. Fuller, Generalized uniserial rings and their Kupisch series, Math. Z. 106 (1968), 248-260. MR 38 \#1118.

4. - On indecomposable injectives over artinian rings, Pacific J. Math. 29 (1969), 115-135. MR $40 \# 186$.

5. M. Harada, QF-3 and semi-primary PP-rings. II, Osaka J. Math. 3 (1966), 21-27. MR 34 \#5874.

6. J. P. Jans, Projective injective modules, Pacific J. Math. 9 (1959), 1103-1108. MR. $22 \# 3750$.

7. T. Kato, Dominant modules, J. Algebra 14 (1970), 341-349. MR 41 \#1792.

8. - Rings of dominant dimension $\geqq 1$, Proc. Japan Acad. 44 (1968), 579-584. MR $38 \# 4525$.

9. - Rings of U-dominant dimension $\geqq 1$, Tôhoku Math。 J. (2) 21 (1969), 321-327. MR 40 \#1423.

10. H. Kupisch, Beiträge zur Theorie nichtalbeinfacher Ringe mit Minimalbedingung, J. Reine Angew。 Math 201 (1959), 100-112. MR 21 \#3460.

11. E. Matlis, Injective modules over noetherian rings, Pacific J. Math。 8 (1958), 511-528. MR $20 \# 5800$.

12. B. J. Müller, On algebras of dominant dimension one, Nagoya Math. J. 31 (1968), 173-183. MR $36 \# 2646$.

13. - The classification of algebras by dominant dimension, Canad. J. Math. 20(1968), 398-409。 MR 37 \#255. 
14. B. J. Müller, Dominant dimension of semi-primary rings, J. Reine Angew. Math 232 (1968), 173-179. MR 38 \#2175.

15. I. Murase, On the structure of generalized uniserial rings. I, Sci. Papers Coll. Gen. Ed. Univ. Tokyo 13 (1963), 1-22. MR 38 \#118.

16. On the structure of generalized uniserial rings. II, Sci. Papers Coll. Gen. Ed. Univ. Tokyo 13 (1963), 131-158. MR 28 \#5086.

17. - On the structure of generalized uniserial rings. III, Sci. Papers Coll. Gen. Ed. Univ. Tokyo 14 (1964), 11-25. MR 31 \#2277.

18. T. Nakayama, On algebras with complete homology, Abh. Math. Sem. Univ. Hamburg 22 (1958), 300-307. MR 21 \# 3471.

19. B. L. Osofsky, A generalization of quasi-Frobenius rings, J. Algebra 4 (1966), 373-387. MR 34 \# 4305.

20. H. Tachikawa, A characterization of QF-3 algebras, Proc. Amer. Math. Soc. 13 (1962), 701-703. MR 26 \# 5027.

21. - On dominant dimensions of QF-3 algebras, Trans. Amer. Math. Soc. 112 (1964), 249-266. MR 28 \#5092.

DEPARTMENT OF MATHEMATICS, VIRGINIA POLYTECHNIC INSTITUTE AND STATE UNIVERSITY, BLACKSBURG, VIRGINIA 24061 